\title{
Hidden Hungers and Hearing Loss in Children
}

\section{Terez BK*}

Faculty of Medicine, Ain Shams University, Egypt

*Corresponding author: Terez BK, Associate Professor of Pediatrics, Faculty of Medicine, Ain Shams University, Cairo, Egypt, E-mail: Terez2003@hotmail.com

\section{Mini Review}

Volume 2 Issue 1

Received Date: March 23, 2017

Published Date: April 04, 2017

DOI: $10.23880 /$ ooaj-16000150

\section{Abstract}

Hidden hungers and Micronutrient deficiencies (MNDs) are global challenges that have a huge impact on health of vulnerable population like children. Nutritional imbalances are increasingly thought to be a causative factor in hearing loss. However, less attention has been paid towards MNDs, which can be prevented. Therefore, this mini-review aims to draw attention of concerned authorities and researchers to combat against MNDs that affect hearing. The major causes of MNDs were poor diet, diseases and infestations, and poor health caring practices. Hearing loss has profound health, social, and economic consequences. Affected children are likely to experience speech, language, and cognitive delays and poor school performance. Only severe prenatal iodine deficiency is listed by the WHO as a nutritional cause of hearing loss, leaving the broad roles of diet and nutrition within its complex set of etiologies yet to be defined.

Keywords: Hearing Loss; MNDS; Protein-energy malnutrition

\section{Introduction}

Apart from the protein-energy malnutrition (PEM, which includes marasmus and kwashiorkor), there exists another form, which is less visible and a result of vitamins and minerals deficiencies, known as micronutrient deficiency (MND) [1]. Therefore, MND can be regarded as a subset of malnutrition. Deficiencies of fat soluble vitamins, iron, and zinc are particularly common, but deficiencies of other water-soluble vitamins, minerals, and trace elements are also found and have great impact in physical, mental, and cognitive development of an individual. Iron deficiency is the most prevalent nutrition problem in the world [2]. Micronutrients (MNs) (vitamins and minerals) are essential for proper growth and development apart from macronutrients (carbohydrates, fats, and proteins). MND has global health impact because its manifestations become less visible and usually begins to show when condition is severe and has already led to serious health burdens, justifying the name "hidden hunger." Deficiencies occur when people do not have access to micronutrient-rich foods such as fruit, vegetables, animal products, and fortified foods, usually because they are too expensive to buy or are locally unavailable [2]. Although the deficiency affects every age group of both sexes, the most vulnerable groups are children and women of reproductive age including pregnant and lactating mothers [3]. The World Health Organization (WHO) estimates that more than 2 billion people are deficient in key vitamins and minerals, including a third of world's children. Most of these people live in low-income countries $[2,4]$.

\section{Hidden Hunger and Hearing Loss}

In fact, Micronutrient deficiencies (MNDs) are increasingly thought to be a causative factor in hearing loss [5]. Age-related hearing loss is actually not due to any kind of mechanical dysfunction in ear; rather it is how brain processes information that results in reduced hearing. Hearing (considered broadly as the function of human auditory brain and its peripheral end organs) has 


\section{Otolaryngology Open Access Journal}

been aligned with other complex neuropsychological processes based on studies of the normal brain and focal brain damage. In the world at large, sounds are embedded in auditory scenes that must be actively deconstructed to identify and track sounds of interest [6]: this, in turn, requires the representation of sound location and movement (auditory spatial analysis) and abstraction of identifying sound characteristics under varying listening conditions (auditory apperceptive processing) [7]. Hearing loss has profound health, social, and economic consequences [8]. Affected children are likely to experience speech, language, and cognitive delays and poor school performance [9]. Major causes of this burden vary across different life stages and include congenital disorders; otitis media; vaccine-preventable infections such as measles, mumps, and rubella; noise exposure; ototoxic drugs; and age [8].

Surprisingly, only severe prenatal iodine deficiency is listed by WHO as a nutritional cause of hearing loss $[2,4,8]$, leaving broad roles of diet and nutrition within its complex set of etiologies still to be defined. In such highincome countries; studies in adults have reported protective roles against hearing loss with higher dietary intakes of fish, long-chain PUFAs, folate, $\beta$-carotene, and vitamins $\mathrm{A}, \mathrm{E}$, and $\mathrm{C}$. Although findings across studies are inconsistent, animal evidence exists to support roles for each of these studied nutrients in regulating redox stress, protecting cochlear function, and enabling hearing. Interestingly, dietary exposure to potentially ototoxic heavy metals (e.g., cadmium, lead), a high BMI and waist circumference (i.e., obesity), and reduced physical activity have also been linked to hearing loss [10,11], which can be attributed by systemic stress of chronically unhealthy diet, lifestyle, and environment that carry consequences for ear health and hearing [11]. Malnutrition continues to be a major public health problem throughout the developing world. About $6 \%$ of children under age of five years are underweight for their age [2,4]. Thus, we must concern about problems caused by malnutrition. It has been validated that nutritional sufficiency especially of protein, iron, choline and long-chain polyunsaturated fatty acids contributes to proper neuronal structure. Deficiency of one or more than one of these elements in the developing brain, due to malnutrition, can lead to impaired myelination, weak synaptic junctions and limited neural arborization [12]. In low-income countries, where under-nutrition remains prevalent, limited research has identified micronutrient deficiencies (e.g., of vitamin A and zinc) as risk factors for otitis media, the leading acquired cause of childhood hearing loss $[13,14]$. Anemia increases risk for auditory dysfunction [14].

\section{Micronutrients that Affect Hearing}

Among micronutrients found to be most beneficial for protecting and improving hearing are: Carotenoids, especially astaxanthin and vitamin A, vitamin C, Folate [15], Zinc [16], and Magnesium [17]. These nutrients support hearing in a number of ways e.g. protecting against oxidative stress inside the cochlea; preventing free radical damage; improving blood flow, thereby reducing cochlear damage related to a compromised vascular system and/ or improving homocysteine metabolism [15-17]. While compelling evidence of the role of vitamin A in preventing hearing loss mediated by ear infection now exists; Experimental animal evidence implicates an essential role for vitamin A in inner ear development so that routinely providing adequate amounts of vitamin A antenatally in undernourished regions of the world may promote normal inner ear development and reduce the risk of sensorineural hearing loss induced by gestational vitamin A deficiency [18].

For noise-induced tinnitus, folate (vitamin B9), has been shown to be beneficial. Folate also lowers homocysteine levels, which have been linked to agerelated hearing loss [15]. Zinc in human plays an important role in cell mediated immunity and is also an antioxidant and anti-inflammatory agent. Zinc supplementation studies in the elderly have shown decreased incidence of infections, decreased oxidative stress, and decreased generation of inflammatory cytokines [19]. Furthermore, research has shown zinc may be useful for sudden idiopathic sensori-neural hearing loss (SSNHL). Whilst, the cause for SSNHL is unknown, one theory is that a viral infection or immunologic disease is involved. Thus may help explain the high rate of recovery, and why zinc appears to be so beneficial for this condition [16]. Zinc has anti-viral properties, and studies have shown it can prevent common cold viruses from replicating or attaching to nasal mucus membranes. Zinc also has immune-boosting properties against viral infection [20].

The nutritional origins of hearing loss differ in chronically undernourished settings [14], where etiologies of premature dysfunction may start in-utero. In India, Choudhury, et al. [21] screened newborn hearing via the auditory brainstem response. Delayed interpeak latencies and wave $\mathrm{V}$ latency, as observed in newborns with latent iron deficiency (i.e., cord plasma serum ferritin $\leq 75 \mathrm{ng} / \mathrm{mL}$ ), is highly suggestive of abnormal myelination of the auditory pathway. Multiple facets of intracellular iron metabolism support oligodendrocytes signaling pathways, proliferation, and formation of myelin, which are critical for auditory neurotransmission 


\section{Otolaryngology Open Access Journal}

[22]. Additionally, Choudhury and his coworkers [21] reinforce the importance of micronutrient imbalance or deficiency as determinants of hearing loss. The authors reported higher intakes of $\beta$-carotene, $\beta$-cryptoxanthin, and folate to be protective against incident hearing loss. Oxidative stress and impaired homocysteine metabolism appear to contribute to inner-ear dysfunction. Also, adequate carotenoid and folate may attenuate risk by postulated scavenging of free radicals and maintenance of antioxidant enzyme homeostasis respectively. Paradoxically, the risk of hearing loss was increased in subjects who reported an increased intake of vitamin C, also an established antioxidant, at amounts that exceeded the Recommended Dietary Allowance (i.e., $>75-90 \mathrm{mg} / \mathrm{d}$ ) but that fell well below the Tolerable Upper Intake Level $(<2000 \mathrm{mg} / \mathrm{d})$ of the Dietary Reference Intakes, suggesting that poorly understood, organ-specific nutrient interactions may exist. They add to a developing story that adequate and balanced nutrition during pregnancy, infancy, and adulthood may reduce hearing loss globally and across life span. Addressing the relation between hidden hungers and hearing loss represents an extraordinary opportunity to explore and potentially reduce the global burden of hearing loss and consequent disability in the developing world [23]. However, it will be imperative to scale up direct nutrition interventions with adequate micronutrients supply, success will be enhanced and sustained by addressing underlying determinants of malnutrition through action in multiple sectors such as poverty alleviation, education, social protection, clean water supply, and sanitation.

\section{References}

1. Sharma M, Atri A (2011) Essentials of International Health. Burlington, Mass, USA: Jones \& Bartlett Learning. Malnutrition, nutritional deficiencies, and obesity: 194 .

2. World Health Organization (2014) Micronutrient Deficiencies: Iron Deficiency Anemia. Geneva, Switzerland: World Health Organization.

3. Thompson B, Amoroso L (2011) Combating Micronutrient Deficiencies: Food-based Approaches. Rome, Italy: Food and Agriculture Organization of the United Nations and CAB International.

4. World Health Organization (2001) Geneva, Switzerland: World Health Organization. The World Health report 2001: reducing risks, promoting healthy life.
5. Sprem N, Branica S, Dawidowsky K (2001) Vasodilator and vitamins in therapy of sensorineural hearing loss following war-related blast injury: retrospective study. Croat Med J 42(6): 646-649.

6. Cope TE, Baguley DM, Griffiths TD (2015) The functional anatomy of central auditory processing. Pract Neurol 15(4): 302-308.

7. Hardy CJ, Marshall CR, Golden HL, Clark CN, Mummery CJ, et al. (2016) Hearing and dementia. J Neurol 263(11): 2339-2354.

8. Olusanya BO, Neumann KJ, Saunders JE (2014) The global burden of disabling hearing impairment: a call to action. Bull World Health Organ 92: 367-73.

9. Emmett SD, Francis HW (2015) The socioeconomic impact of hearing loss in U.S. adults. Otol Neurotol 36: 545-550.

10. Shargorodsky J, Curhan SG, Henderson E, Eavey R, Curhan GC (2011) Heavy metals exposure and hearing loss in US adolescents. Arch Otolaryngol Head Neck Surg 137(12): 1183-1189.

11. Hwang JH, Wu CC, Hsu CJ, Liu TC, Yang WS (2009) Association of central obesity with the severity and audiometric configurations of age-related hearing impairment. Obesity (Silver Spring) 17(9): 17961801.

12. Bourre JM Effects of nutrients (in food) on the structure and function of the nervous system: update on dietary requirements for brain. Part 1: micronutrients. J Nutr Health Aging 10(5): 377-385.

13. Elemraid MA, Mackenzie IJ, Fraser WD, Brabin BJ (2009) Nutritional factors in the pathogenesis of ear disease in children: a systematic review. Ann Trop Paediatr 29(2): 85-99.

14. Kamel TB, Deraz TE, Elkabarity RH, Ahmed RK (2016) Protein energy malnutrition associates with different types of hearing impairments in toddlers: Anemia increases cochlear dysfunction. Int $\mathrm{J}$ Pediatr Otorhinolaryngol 85: 27-31.

15. Curhan SG, Stankovic KM, Eavey RD, Wang M, Stampfer MJ, et al. (2015) Carotenoids, vitamin A, vitamin $\mathrm{C}$, vitamin $\mathrm{E}$, and folate and risk of selfreported hearing loss in women. Am J Clin Nutr 102(5): 1167-1175. 


\section{Otolaryngology Open Access Journal}

16. Yang CH, Ko MT, Peng JP, Hwang CF (2011) Zinc in the treatment of idiopathic sudden sensorineural hearing loss. Laryngoscope 121(3): 617-621.

17. Jiao J, Gu GZ, Chen GS, Zheng YX, Zhang HL, et al. (2016) Association between serum magnesium ion level and risk of noise-induced hearing loss. Zhonghua Lao Dong Wei Sheng Zhi Ye Bing Za Zhi 34(12): 884-888.

18. Emmett SD, West KP (2014) Gestational vitamin A deficiency: a novel cause of sensorineural hearing loss in the developing world? Med Hypotheses 82(1): 6-10.

19. Prasad AS (2014) Zinc: an antioxidant and antiinflammatory agent: role of zinc in degenerative disorders of aging. J Trace Elem Med Biol 28(4): 364371.
20. Allan GM, Arroll B (2014) Prevention and treatment of the common cold: making sense of the evidence. CMAJ 186(3): 190-199.

21. Choudhury V, Amin SB, Agarwal A, Srivastava LM, Soni A, et al. (2015) Latent iron deficiency at birth influences auditory neural maturation in late preterm and term infants. Am J Clin Nutr 102(5): 1030-1034.

22. Bakoyiannis I, Gkioka E, Daskalopoulou A, Korou LM, Perrea D, et al. (2015) An explanation of the pathophysiology of adverse neurodevelopmental outcomes in iron deficiency. Rev Neurosci 26(4): 479488.

23. Olusanya BO, Luxon LM, Wirz SL (2005) Childhood deafness poses problems in developing countries. BMJ 330(7489): 480-481. 